\title{
Variability of alder-dominated forest vegetation along a latitudinal gradient in Slovakia
}

\author{
Michal Slezák ${ }^{1,2 *}$, Richard Hrivnák², Anna Petrášová3 ${ }^{3}$ Daniel Dítě ${ }^{2}$ \\ ${ }^{1}$ Department of Biology and Ecology, Catholic University, Hrabovská cesta 1, 03401 Ružomberok, Slovakia \\ ${ }^{2}$ Institute of Botany, Slovak Academy of Sciences, Dúbravská cesta 9, 84523 Bratislava, Slovakia \\ ${ }^{3}$ Department of Biology and Ecology, Matej Bel University, Tajovského 40, 97401 Banská Bystrica, Slovakia
}

\begin{abstract}
Syntaxonmical revision of azonal forest vegetation with dominance of Alnus glutinosa and A. incana was done along a latitudinal transect of Slovakia. A data matrix consisting of 240 phytosociological relevés was obtained in accordance with the standard Zürich-Montpellier approach. Detrended correspondence analysis was used to clarify the relationships between the vegetation composition and environmental variables, whereas one-way ANOVA was applied to quantify the differences in site requirements of particular vegetation types. The unsupervised numerical classification resulted in identification of five clusters corresponding to the traditionally described and ecologically interpretable associations within the Euro-Siberian alder carr forests of Alnion glutinosae and the European broad-leaved floodplain forests of Alnion incanae: Carici elongatae-Alnetum glutinosae Schwickerath 1933 (alder carr forests on permanently waterlogged soils), Stellario-Alnetum glutinosae Lohmeyer 1957 (riparian alder vegetation on mesic to humid sites along small brooks), Piceo-Alnetum Mráz 1959 (submontane and montane oligotrophic spruce-alder forests on waterlogged habitats), Cardamino amarae-Alnetum incanae Šomšák 1961 (grey alder vegetation in spring fed areas) and Alnetum incanae Lüdi 1921 (submontane and montane streamside grey alder forests on mesic sites). They significantly $(P<0.05)$ differed in the Ellenberg's indicator values for nutrients, moisture, temperature and altitude. These environmental variables were also established by DCA analysis as underlying sources of variation in alder-dominated forest composition. Special attention was given to discussion of their syntaxonomy, nomenclature, floristic structure, ecological features and distribution.
\end{abstract}

Keywords: syntaxonomy, Alnus glutinosa, Alnus incana, numerical classification, nomenclature

\section{Introduction}

Plant species establishment and survival along environmental gradients have commonly been attributed to different environmental niches, life-history and adaptive traits [1]. Effective phenotype adaptation of alder species to extreme soil conditions allows them to form canopy-closed forest vegetation on alluvial and marshland localities. Alder forests represent azonal plant communities developed independently of the variables determining vegetation zonality. The hydrological regime was most often identified as the underlying source of variation in their floristic spectrum. Seasonal dynamic of groundwater table and flood regime are important ecological phenomenon creating microhabitats with various environmental qualities [2-5].

*Corresponding author. Email: slezak.miso@gmail.com Handling Editor: Zygmunt Kącki

This is an Open Access digital version of the article distributed under the terms of the Creative Commons Attribution 3.0 License (creativecommons.org/licenses/by/3.0/), which permits redistribution, commercial and non-commercial, provided that the article is properly cited.
The diverse ecological requirements and distribution patterns of black alder (Alnus glutinosa) and grey alder (Alnus incana) [6,7], considered as native species to Central Europe, result in various syntaxonomical positions of their stands. Alder-dominated forests on permanently waterlogged soils are traditionally assigned to the Euro-Siberian alder carr forests of the Alnion glutinosae alliance (Alnetea glutinosae class), whereas the more mesophilous streamside forests are recorded within the European broad-leaved floodplain forests of the Alnion incanae (= Alno-Ulmion) alliance (Querco-Fagetea class). These ecologically and floristically distinctive vegetation types are scattered relatively evenly all over the Central European landscape [3,8-12]. The presented classification concept belongs to the widely accepted and frequently used in vegetation surveys, namely in Poland [13], Ukraine [14], Hungary [15], Austria [16], the Czech Republic [4], Germany [17] and also in Slovakia [18]. On the other hand, there is a considerable inconsistency in internal differentiation of these two alliances resulting probably from diverse level of variability in their species composition. While the higher number of associations has been distinguished in the Alnion incanae forests (e.g. $[4,19])$, the opposite pattern has been reported from the Alnion glutinosae communities $[11,16]$.

The Slovak phytosociological data set contains especially relevés from local vegetation studies performed in lower mountains 
$[20,21]$, lowlands, basins or foothills of the Western Carpathians [22-24]. Their results are mostly only of local validity because they concerned small areas. Preliminary syntheses of selected alder vegetation types at national scale using non-formalized (expert-based) approach $[25,26]$ belong to other important resources of accessible data. Our paper is thus intended to covering the overall vegetation variation in alder-dominated forests in wider geographical area, using a more formalized classification method. The north-south (latitudinal) transect across central Slovakia (Fig. 1), which was chosen for this purpose, represents also the whole altitudinal gradient reported for alder forests in Slovakia. In more detail, the original and earlier published relevés have been collected from the planar to the montane belt in the Carpathian and Pannonian biogeographical regions. In general, the species variation and spatial distribution of alder forests, and their syntaxonomical interpretation remain still vague in Slovakia. Therefore, the aim of this paper is: $(\boldsymbol{i})$ to establish the main alder-dominated vegetation units along the present latitudinal transect and to propose their syntaxonomical scheme, and (ii) to determine environmental factors affecting the variation in alder forest composition.

\section{Material and methods}

There was studied forest vegetation with dominance (at least $25 \%$ cover) of Alnus glutinosa and/or A. incana in the tree layer along a north-south transect of Slovakia (Fig. 1). The vegetation data matrix involved only phytosociological relevés with a plot size of $200-450 \mathrm{~m}^{2}$ and identified moss layer. Subsequently, the relevés with a tree layer cover below the $60 \%$ were excluded from the further analysis. The final data set of 240 relevés thus included 101 unpublished relevés collected by the authors in 2009-2011 and 139 relevés stored in the "Slovak phytosociological database" [27] or excerpted from the literature sources. The research was carried out in accordance with the principles of the Zürich-Montpellier approach [28], mostly using the modified nine-degree Braun-Blanquet sampling scale $(\mathrm{r},+, 1,2 \mathrm{~m}, 2 \mathrm{a}$, $2 b, 3,4,5)$ [29]. Every species observation was assigned to a vertical stratum (E0 - moss layer, E1 - herb layer, E2 - shrub layer and E3 - tree layer) [28].

The numerical classification was performed in the PC-ORD program [30] following the Relative Manhattan's distance as a measure of dissimilarity, the Beta flexible linkage method with coefficient $\beta=-0.25$ and the logarithmic data transformation. The optimal number of clusters was determined based on the "crispness" procedure [31] in the Juice program [32]. Several taxa were merged to aggregates (agg.), broadly defined taxa (s. lat.), genus (sp. div.) or species level: Aconitum firmum (incl. A. firumum subsp. firmum, A. firmum subsp. moravicum), Agrostis stolonifera agg. (A. gigantea, A. stolonifera), Alchemilla sp. div. (A. monticola, A. vulgaris agg., A. xanthochlora), Caltha palustris (C. palustris subsp. laeta, C. palustris subsp. palustris), Cardamine amara agg. (C. amara, C. amara subsp. opicii), Dactylis glomerata agg. (D. glomerata, D. polygama), Dactylorhiza majalis s. lat. (D. fuchsii, D. maculata, D. majalis), Dryopteris carthusiana agg. (D. carthusiana, D. dilatata), Galeobdolon luteum agg. (G. luteum, G. montanum), Glechoma hederacea agg. (G. hederacea, G. hirsuta), Molinia caerulea agg. (M. arundinacea, M. caerulea), Myosotis scorpioides agg. ( $M$. caespitosa, M. laxiflora, M. scorpioides), Plagiomnium affine s. lat. (P. affine, P. elatum, P. ellipticum), Ranunculus auricomus agg. (R. cassubicus s. lat.), Rubus fruticosus agg. (R. hirtus s. lat.), Senecio nemorensis agg. (S. germanicus, S. ovatus), Oxycoccus palustris agg. (O. microcarpus, O. palustris), Valeriana dioica agg. (V. dioica, V. simplicifolia) and Valeriana officinalis agg. (V. excelsa subsp. sambucifolia, V. officinalis). The same species recorded in different layers were merged into one layer for the purpose of numerical classification. In order to confirm our results of classification and to achieve better syntaxonomical interpretation of particular clusters, the data set extended by phytosociological relevés of original diagnosis of detected vegetation units [20,33-36] was analyzed repeatedly.

The diagnostic species in the target group of relevés were identified according to the concept of fidelity ( $\Phi$ - phi coefficient) [37], frequency and constancy (difference of constancy class, i.e. 20\%). All relevé groups were standardized to an equal size, and fidelity calculation was done using the presence/absence data. Threshold values of phi coefficient and frequency for the species to be considered as diagnostic were set to $\Phi>0.30$ and $30 \%$, respectively. The species with probability of random occurrence in the vegetation type determined by Fisher's exact test $(P \geq 0.01)$ were eliminated from the list of diagnostic taxa.

Major variation patterns in species composition were assessed using the unimodal ordination technique (detrended correspondence analysis; DCA). The species cover values recorded on the Braun-Blanquet scale were replaced by midpercentage values for each degree and transformed logarithmically. For interpretation of the main environmental gradients, altitude and non-weighted Ellenberg indicator values (EIV) of vascular plants for light, temperature, continentality, moisture, soil reaction and nutrients [38] were calculated for relevés and
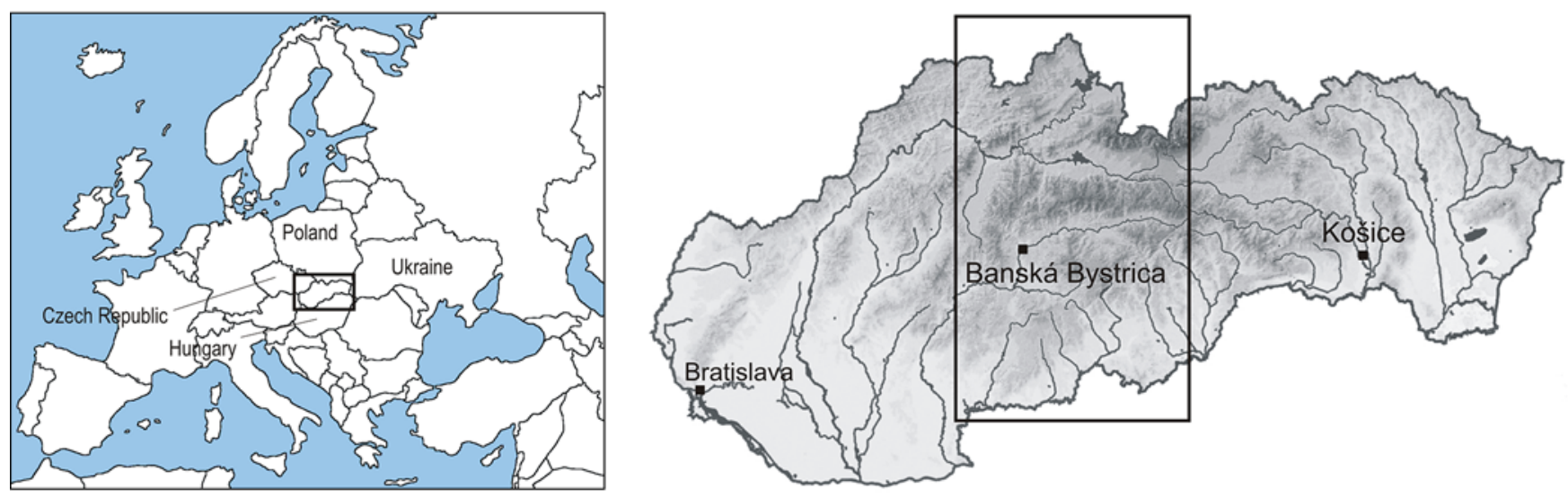

Fig. 1 Map of the study area in Central Europe. 
plotted into the DCA ordination diagram as the supplementary variables. The Pearson correlation coefficient was applied to determine the importance of explanatory variables for changes in the community structure when their values were correlated with the relevés scores on the first two ordination axes. The normal distribution of environmental parameters was tested with the Shapiro-Wilk W test and the homogeneity of variance with the Levene's test. To ensure normality of distribution and to increase linearity of response, the explanatory variable of EIV for nutrients was transformed biquadratically prior to the analysis. The pairwise differences in altitude and EIV among vegetation units were tested by the one-way ANOVA and post-hoc Tukey HSD test. The transformed variables showing still left- or right-skewed trend in the values distribution were analyzed by multiple comparisons using the non-parametric KruskalWallis ANOVA. All the statistical analyses were computed in the Statistica program (StatSoft Inc., Tulsa, OK, USA) and Canoco for Windows package (Microcomputer Power, Ithaca, NY, US). The altitude was measured with Garmin GPSmap 60 CSx equipment or taken from literature. The altitudinal range was between 165 and $1025 \mathrm{~m}$ a.s.l.

The nomenclature of vascular plants is in accordance with the checklist by Marhold \& Hindák [39] and bryophytes with the papers of [40] and [41]. The names of plant communities follow [18], and in the syntaxonomical scheme they are presented with the author's name and year of description. The nomenclatural revision of the particular vegetation units frequently used in the study area was done according to the rules of the International Code of Phytosociological Nomenclature (ICPN) [42].

\section{Results}

\section{Species composition pattern}

Altogether 571 plant species including 434 vascular plants and 137 bryophytes (28 liverworts and 109 mosses) was recorded in 240 phytosociological relevés. The most common species of the alder-dominated forests documented in more than $60 \%$ plots were mainly the wet sites generalists accompanied by nutrient-demanding species (Alnus glutinosa, Athyrium filix-femina, Caltha palustris, Crepis paludosa, Deschampsia cespitosa, Dryopteris carthusiana agg., Filipendula ulmaria, Ranunculus repens, Rubus idaeus and Urtica dioica; Tab. 1). The DCA analysis showed the general trends in floristic variation (Fig. 2). The species scores in ordination space indicated a pronounced shift from plants showing affinity to eutrophic habitats at lower altitudes (e.g. Acer campestre, Circaea lutetiana, Geum urbanum) to mesotrophic and slightly oligotrophic species of submontane and montane sites (Dicranum scoparium, Equisetum sylvaticum, Picea abies). Along the second axis, a course from forest species adapted to mesophilous substrate (Asarum europaeum, Geranium robertianum, Primula elatior) to marsh and spring taxa (Cardamine amara agg., Galium palustre, Lycopus europaeus) was evident (Fig. 2).

The numerical classification resulted in delimitation of five floristically well identified clusters (Tab. 1):

CLUSTER 1 . These alder carr forests are exclusively dominated by Alnus glutinosa. Shade-tolerant species Frangula alnus and Padus avium are usually present in the shrub layer. To the most frequent dominants of the understorey belong Caltha palustris and Carex acutiformis, although other wet nitrophilous species (e.g. Filipendula ulmaria, Impatiens noli-tangere,

Tab. 1 Synoptic table of alder forests with frequencies and fidelities (phi coefficient $\times 100$ in the upper indices) for Alnus-dominated forest vegetation from central Slovakia.

\begin{tabular}{lccccc} 
Number of cluster & 1 & 2 & 3 & 4 & 5 \\
Number of relevés & 49 & 70 & 38 & 48 & 35 \\
\hline
\end{tabular}

E3

$\begin{array}{lccccc}\text { Alnus glutinosa } & 100^{40.1} & 91^{31.3} & 95^{34.7} & 12^{--} & 6^{--} \\ \text {Alnus incana } & 12^{--} & 17^{--} & 50^{--} & 98^{42.7} & 100^{44.8} \\ \text { Picea abies } & 2^{--} & 3^{--} & 97^{55.7} & 75^{33.1} & 34^{-\cdots}\end{array}$

E2

Picea abies

Alnus incana

Padus avium

Frangula alnus

E1

Diagnostic taxa of cluster 1

Carex elongata

Lycopus europaeus

Lythrum salicaria

Carex acutiformis

Scutellaria galericulata

Solanum dulcamara

Galium palustre

Scirpus sylvaticus

Equisetum fluviatile

Calliergonella cuspidata (E0)

Juncus effusus

Diagnostic taxa of cluster 2

Geum urbanum

Sambucus nigra (E2)

Circaea lutetiana

Acer campestre (E2)

Acer campestre

Euonymus europaeus

Galium aparine

Brachypodium sylvaticum

Glechoma hederacea agg.

Galeobdolon luteum agg.

Corylus avellana (E2)

Diagnostic taxa of cluster 3

Luzula pilosa

Calamagrostis villosa

Stellaria alsine

Vaccinium myrtillus

Sphagnum centrale (E0)

Dicranum scoparium (E0)

Vaccinium vitis-idaea

Calypogeia integristipula (E0)

Betula pubescens (E3)

Chiloscyphus polyanthos (E0)

Pellia sp. (E0)

Carex echinata

Picea abies

Galium uliginosum

$\begin{array}{ccccc}10^{--} & 4^{--} & 89^{42.8} & 73^{26.2} & 57^{--} \\ 14^{--} & 3^{--} & 39^{--} & 71^{33.7} & 63^{25.5} \\ 51^{22.5} & 41^{--} & 5^{--} & 8^{--} & 46^{--} \\ 33^{20.0} & 10^{--} & 34^{22.1} & 10^{--} & .-\end{array}$

$69^{72 .}$

$82^{57.8}$

$45^{53.2}$

$35^{51.6}$

$33^{51.3}$

$71^{45.2}$

$73^{45.2}$

$71^{44.4}$

$41^{34.9}$

$45^{33.8}$

$49^{32.5}$

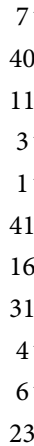

$40^{--}$

8

1

$3^{---}$

$1^{---}$

$41^{--}$

$16^{--}$

$31^{-\cdots}$

$4^{---}$

$6^{---}$

$23^{--}$

\begin{tabular}{|c|c|}
\hline $4^{--}$ &.$\cdots$ \\
\hline $10^{---}$ & $6^{-\cdots}$ \\
\hline.$\cdots$ &.$\cdots$ \\
\hline.$\cdots$ &.-- \\
\hline.$^{-\cdots}$ &.$\cdots$ \\
\hline $4^{--}$ & $17^{-}$ \\
\hline $25^{--}$ & 14 \\
\hline $27^{--}$ & 20 \\
\hline $8^{---}$ & $6^{-\cdots}$ \\
\hline $12^{---}$ & $11^{-}$ \\
\hline $15^{--}$ & $3^{-\cdots}$ \\
\hline
\end{tabular}

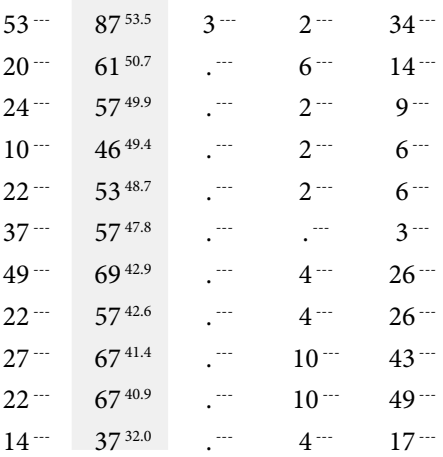

$34^{--}$
$14^{--}$
$9^{--}$
$6^{--}$
$6^{--}$
$3^{-\cdots}$
$26^{--}$
$26^{-}$
$43^{-}$
$49^{--}$
$17^{-}$

$63^{67.0}$

$63^{57.6} 29^{13}$

$45^{57.1} \quad 2^{---}$

$68^{55.4} \quad 35^{15.7}$

$42^{54.9}$

$71^{52.3}$

$46^{23.3}$

$42^{50.0} \quad 12$

$34^{49.8}$

$37^{48.5}$

$32^{48.1}$

$34^{47.9}$

$39^{47.8}$

$92^{47.0}$

$2^{-\cdots} \quad \cdot^{-\cdots} \quad 34^{46.1}$ 
Tab. 1 (continued)

\begin{tabular}{|c|c|c|c|c|c|}
\hline Number of cluster & 1 & 2 & 3 & 4 & 5 \\
\hline Number of relevés & 49 & 70 & 38 & 48 & 35 \\
\hline Rhytidiadelphus triquetrus (E0) & $\cdot-\cdot$ & $1^{\cdots-}$ & $53^{44.8}$ & $25^{--}$ & $11^{\cdots}$ \\
\hline Alnus glutinosa & $29^{---}$ & $26^{--}$ & $53^{38.1}$ &.$^{---}$ & $\cdot-\cdot$ \\
\hline Agrostis stolonifera agg. & $29^{--}$ & $26^{--}$ & $63^{37.4}$ & $8^{--}$ & $20^{--}$ \\
\hline Alnus glutinosa (E2) & $27^{--}$ & $26^{--}$ & $50^{35.1}$ & $4^{--}$ &.-- \\
\hline Climacium dendroides (E0) & $31^{--}$ & $11^{--}$ & $68^{33.7}$ & $35^{--}$ & $34^{--}$ \\
\hline \multicolumn{6}{|l|}{ Diagnostic taxa of cluster 4} \\
\hline Gentiana asclepiadea &.$^{---}$ &.$^{---}$ &.$^{---}$ & $31^{43.0}$ & $9^{--}$ \\
\hline Equisetum palustre & $24^{--}$ & $7^{--}$ & $18^{--}$ & $54^{34.9}$ & $17^{--}$ \\
\hline \multicolumn{6}{|l|}{ Diagnostic taxa of cluster 5} \\
\hline Primula elatior & $8^{--}$ & $19^{--}$ & $5^{--}$ & $35^{--}$ & $86^{59.8}$ \\
\hline Petasites hybridus & $4^{--}$ & $9^{---}$ & $3^{--}$ & $25^{---}$ & $71^{58.9}$ \\
\hline Dactylis glomerata agg. & $4^{--}$ & $21^{--}$ & $3^{--}$ & $12^{---}$ & $66^{54.3}$ \\
\hline Valeriana officinalis agg. & $16^{--}$ & $14^{--}$ & $5^{--}$ & $27^{--}$ & $74^{52.5}$ \\
\hline Asarum europaeum & $4^{--}$ & $47^{14.7}$ & $\cdot-\cdot$ & $35^{---}$ & $80^{49.5}$ \\
\hline Astrantia major & $4^{--}$ & $7^{---}$ &.$^{---}$ & $\cdot-\cdot$ & $40^{49.1}$ \\
\hline Aconitum variegatum &.$^{---}$ &.$^{---}$ & $3^{--}$ &.$^{---}$ & $31^{48.9}$ \\
\hline Roegneria canina & $16^{---}$ & $27^{--}$ & $3^{--}$ & $4^{--}$ & $63^{48.1}$ \\
\hline Plagiomnium undulatum (E0) & $24^{--}$ & $54^{--}$ & $21^{--}$ & $31^{\cdots-}$ & $89^{45.0}$ \\
\hline Silene dioica &.$^{---}$ & $3^{--}$ & $\cdot-\cdot$ & $15^{\cdots-}$ & $40^{44.7}$ \\
\hline Geum rivale & $27^{--}$ & $21^{--}$ & $34^{--}$ & $69^{20.3}$ & $91^{43.0}$ \\
\hline Ranunculus lanuginosus & $2^{--}$ & $21^{--}$ &.$^{---}$ & $31^{\cdots-}$ & $57^{41.7}$ \\
\hline Daphne mezereum & $\cdot-\cdot$ & $3^{\cdots}$ & $3^{--}$ & $23^{--}$ & $43^{40.9}$ \\
\hline Poa nemoralis &.$^{---}$ & $7^{--}$ & $3^{--}$ & $6^{--}$ & $34^{40.3}$ \\
\hline Galeopsis pubescens & $4^{--}$ & $7^{---}$ & $\cdot-\cdot$ & $6^{--}$ & $31^{36.4}$ \\
\hline Angelica sylvestris & $39^{---}$ & $19^{--}$ & $11^{--}$ & $35^{--}$ & $69^{36.0}$ \\
\hline Heracleum sphondylium & $2^{-\cdots}$ & $13^{--}$ & $\cdot^{---}$ & $8^{--}$ & $34^{35.7}$ \\
\hline Geranium phaeum &.$^{---}$ & $17^{--}$ &.$^{---}$ & $12^{--}$ & $37^{35.0}$ \\
\hline Carduus personata & $6^{--}$ & $11^{--}$ & $3^{--}$ & $17^{---}$ & $40^{34.1}$ \\
\hline Acer pseudoplatanus (E2) & $14^{---}$ & $17^{--}$ &.$^{---}$ & $4^{---}$ & $37^{32.0}$ \\
\hline
\end{tabular}

\section{Other species}

\section{Caltha palustris}

Urtica dioica

Filipendula ulmaria

Rubus idaeus

Athyrium filix-femina

Dryopteris carthusiana agg.

Ranunculus repens

Crepis paludosa

Deschampsia cespitosa

Myosotis scorpioides agg.

Chaerophyllum hirsutum

Cardamine amara agg.

Impatiens noli-tangere

Lysimachia vulgaris

Chrysosplenium alternifolium

Oxalis acetosella

Aegopodium podagraria

Stellaria nemorum

Stachys sylvatica

Cirsium oleraceum

Senecio nemorensis agg.

Valeriana dioica agg.

Ajuga reptans

Festuca gigantea

Carex remota

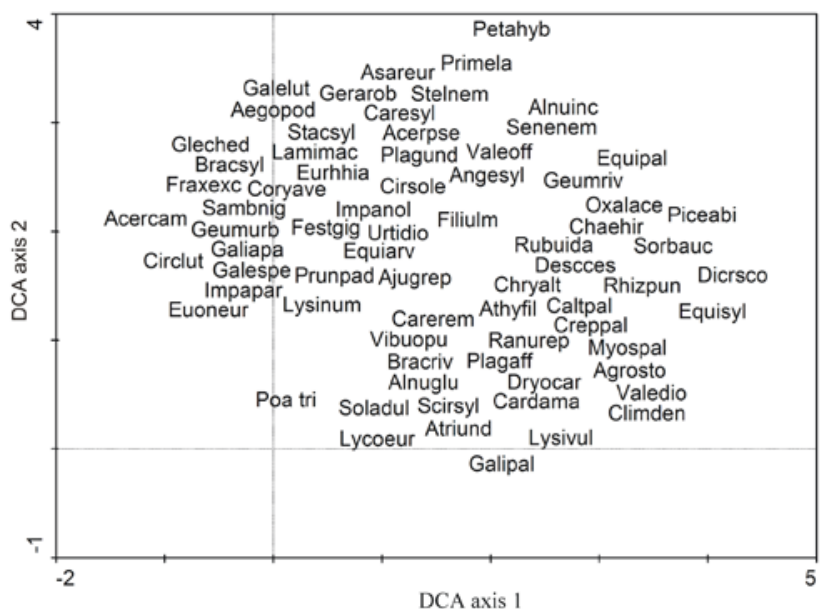

Fig. 2 Species scatter plot of alder forest vegetation obtained by DCA ordination. Acercam - Acer campestre; Acerpse - Acer pseudoplatanus; Aegopod - Aegopodium podagraria; Ajugrep - Ajuga reptans; Alnuglu - Alnus glutinosa; Alnuinc - Alnus incana; Angesyl - Angelica sylvestris; Agrosto - Agrostis stolonifera agg.; Asareur - Asarum europaeum; Athyfil - Athyrium filix-femina; Atriund - Atrichum undulatum; Bracriv - Brachythecium rivulare; Bracsyl - Brachypodium sylvaticum; Caltpal - Caltha palustris; Cardama - Cardamine amara agg.; Carerem - Carex remota; Caresyl - Carex sylvatica; Chaehir - Chaerophyllum hirsutum; Chryalt - Chrysosplenium alternifolium; Circlut - Circaea lutetiana; Cirsole - Cirsium oleraceum; Climden - Climacium dendroides; Coryave - Corylus avellana; Creppal - Crepis paludosa; Descces - Deschampsia cespitosa; Dicrsco - Dicranum scoparium; Dryocar - Dryopteris carthusiana agg.; Equiarv - Equisetum arvense; Equipal - Equisetum palustre; Equisyl - Equisetum sylvaticum; Euoneur - Euonymus europaeus; Eurhhia - Eurhynchium hians; Festgig - Festuca gigantea; Filiulm - Filipendula ulmaria; Fraxexc - Fraxinus excelsior; Galelut - Galeobdolon luteum agg.; Galespe - Galeopsis speciosa; Galiapa - Galium aparine; Galipal - Galium palustre; Gerarob Geranium robertianum; Geumriv - Geum rivale; Geumurb - Geum urbanum; Gleched - Glechoma hederacea agg.; Impanol - Impatiens noli-tangere; Impapar - Impatiens parviflora; Lamimac - Lamium maculatum; Lycoeur - Lycopus europaeus; Lysinum - Lysimachia nummularia; Lysivul - Lysimachia vulgaris; Myospal - Myosotis scorpioides agg.; Oxalace - Oxalis acetosella; Petahyb - Petasites hybridus; Piceabi - Picea abies; Plagaff - Plagiomnium affine s. lat.; Plagund - Plagiomnium undulatum; Poatri - Poa trivialis; Primela - Primula elatior; Prunpad - Padus avium; Ranurep - Ranunculus repens; Rhizpun Rhizomnium punctatum; Rubuida - Rubus idaeus; Sambnig - Sambucus nigra; Scirsyl - Scirpus sylvaticus; Senenem - Senecio nemorensis agg.; Soladul - Solanum dulcamara; Sorbauc - Sorbus aucuparia; Stacsyl - Stachys sylvatica; Stelnem - Stellaria nemorum; Urtidio - Urtica dioica; Valedio - Valeriana dioica agg.; Valeoff - Valeriana officinalis agg.; Vibuopu - Viburnum opulus.

Persicaria hydropiper, Scirpus sylvaticus) and/or spring plants (Cardamine amara agg., Crepis paludosa) can also be found with a higher cover. This vegetation is well differentiated by the presence of species adapted to waterlogged hollows (Equisetum fluviatile, Galium palustre, Lycopus europaeus, Scutellaria galericulata) along with hygrophilous elements showing affinity to hummocks (Carex elongata, Dryopteris carthusiana agg.). They are accompanied by plants of wet meadows (Cirsium oleraceum, Lysimachia vulgaris, Lythrum salicaria, Poa trivialis) and liana species (Humulus lupulus, Solanum dulcamara). The regular pattern of community structure is species-rich moss layer 
Tab. 1 (continued)

\begin{tabular}{|c|c|c|c|c|c|}
\hline Number of cluster & 1 & 2 & 3 & 4 & 5 \\
\hline Number of relevés & 49 & 70 & 38 & 48 & 35 \\
\hline Lysimachia nummularia & $57^{29.3}$ & $56^{27.8}$ & $3^{--}$ & $4^{--}$ & $31^{--}$ \\
\hline Lamium maculatum & $14^{--}$ & $57^{28.9}$ &.-- & $27^{\cdots-}$ & $54^{25.8}$ \\
\hline Viburnum opulus & $59^{34.8}$ & $40^{--}$ & $16^{--}$ & $10^{---}$ & $14^{--}$ \\
\hline Poa trivialis & $65^{45.9}$ & $51^{29.9}$ & $5^{--}$ & $2^{--}$ & $3^{--}$ \\
\hline Equisetum sylvaticum & $8^{--}$ & $3^{\cdots-}$ & $74^{42.0}$ & $56^{23.6}$ & $29--$ \\
\hline Galeopsis speciosa & $43^{--}$ & $56^{35.1}$ &.-- & $10^{--}$ & $17^{\cdots-}$ \\
\hline Acer pseudoplatanus & $31^{--}$ & $30^{--}$ & $\cdot-\cdot$ & $31^{\cdots}$ & $54^{27.5}$ \\
\hline Geranium robertianum & $12^{--}$ & $47^{20.8}$ &.-- & $17^{--}$ & $66^{41.4}$ \\
\hline Padus avium & $45^{19.6}$ & $39^{--}$ & $13^{---}$ & $6^{--}$ & $34^{--}$ \\
\hline Alnus incana & $4^{--}$ & $9^{--}$ & $13^{--}$ & $62^{33.5}$ & $69^{40.1}$ \\
\hline Equisetum arvense & $35^{--}$ & $40^{15.6}$ & $3^{--}$ & $8^{--}$ & $46^{22.1}$ \\
\hline Sorbus aucuparia & $20^{--}$ & $9^{---}$ & $53^{25.0}$ & $42^{--}$ & $26^{--}$ \\
\hline Fraxinus excelsior & $39^{---}$ & $56^{41.4}$ & $\cdot-\cdots$ & $2^{--}$ & $11^{--}$ \\
\hline Carex sylvatica & $8^{--}$ & $40^{19.3}$ & $5^{--}$ & $19^{---}$ & $46^{26.1}$ \\
\hline Impatiens parviflora & $33^{--}$ & $43^{28.8}$ & $11^{--}$ & $10^{--}$ & $3^{--}$ \\
\hline Rumex obtusifolius & $39^{22.3}$ & $24^{--}$ & $3^{--}$ & $21^{---}$ & $17^{--}$ \\
\hline Milium effusum & $10^{--}$ & $23^{--}$ &.$-\cdot$ & $29^{--}$ & $43^{26.8}$ \\
\hline Persicaria hydropiper & $49^{42.7}$ & $36^{25.0}$ &.-- &.-- & $\cdot-\cdot$ \\
\hline Maianthemum bifolium & $12^{--}$ & $1^{--}$ & $42^{22.3}$ & $29^{---}$ & $31^{---}$ \\
\hline Paris quadrifolia & $8^{-\cdots}$ & $10^{--}$ & $13^{--}$ & $40^{25.7}$ & $26^{\cdots-}$ \\
\hline Rubus fruticosus agg. & $31^{23.8}$ & $37^{33.2}$ & $3^{--}$ &.$\cdots$ &.$^{---}$ \\
\hline Symphytum tuberosum & $6^{--}$ & $36^{30.1}$ & $\cdot-\cdot$ & $2^{--}$ & $29^{---}$ \\
\hline Humulus lupulus & $39^{37.8}$ & $27^{20.6}$ & $\cdot \cdot-\cdot$ & $\cdot^{---}$ & $\cdot-\cdot$ \\
\hline \multicolumn{6}{|l|}{ E0 } \\
\hline Brachythecium rivulare & $82^{31.4}$ & $70^{19.7}$ & $39^{--}$ & $15^{--}$ & $46^{--}$ \\
\hline Plagiomnium affine s.l. & $63^{19.8}$ & $33^{--}$ & $32^{--}$ & $33^{--}$ & $57^{\cdots-}$ \\
\hline Rhizomnium punctatum & $20^{--}$ & $26^{--}$ & $58^{25.2}$ & $38^{--}$ & $29^{--}$ \\
\hline Atrichum undulatum & $49^{24.6}$ & $33^{--}$ & $26^{--}$ & $10^{--}$ & $17^{\cdots-}$ \\
\hline Oxyrrhynchium hians & $14^{--}$ & $37^{20.8}$ &.$^{---}$ & $10^{\cdots-}$ & $40^{24.4}$ \\
\hline Polytrichastrum formosum & $14^{--}$ & $1^{--}$ & $42^{29.0}$ & $35^{20.6}$ & $3^{--}$ \\
\hline
\end{tabular}

Each column represents an association: 1 - Carici elongatae-Alnetum glutinosae s. lat.; 2 - Stellario-Alnetum glutinosae; 3 - Piceo-Alnetum; 4 - Cardamino amarae-Alnetum incanae; 5 - Alnetum incanae. Diagnostic species of communities are shaded and ranked by decreasing phi values. Species with frequency $<10 \%$ (in total 447 species) were omitted from the table. Sources: CL1: original unpublished - 37 relevés; Kliment \& Wazka [21] - 3 rel.; Slezák et al. [24] - 9 rel.; CL2: original unpublished - 44 rel.; Hrivnák \& Cvachová [56] - 1 rel.; Hrivnák et al. [57] - 2 rel.; Kliment \& Wazka [21] - 1 rel.; Miadok [58] - 1 rel.; Slezák et al. [24] - 19 rel.; Slezák et al. [59] - 1 rel.; Watzka [60] - 1 rel.; CL3: original unpublished - 1 rel.; Ferančíková [61] - 1 rel.; Holotová [62] - 3 rel.; Matová [63] - 4 rel.; Šomšák [50] - 8 rel.; Viceníková [64] - 21 rel.; CL4: original unpublished - 6 rel.; Černušáková [65] - 16 rel.; Ferančíková [61] - 1 rel.; Holotová [62] - 4 rel.; Hrivnák et al. [57] - 1 rel.; Jurko [25] - 5 rel.; Kubíček et al. [66] - 1 rel.; Šomšák [67] - 1 rel.; Šomšák et al. [68] - 4 rel.; Viceníková [64] - 7 rel.; Watzka [60] - 2 rel.; CL5: original unpublished - 13 rel.; Hrivnák \& Cvachová [56] - 1 rel.; Hrivnák et al. [57] - 1 rel.; Jurko [25] - 9 rel.; Jurko \& Májovský [69] - 6 rel.; Kanka [70] - 3 rel.; Kučera [71] - 1 rel.; Viceníková [64] - 1 rel. with common species of wet habitats (Atrichum undulatum, Brachythecium rivulare, Calliergonella cuspidata, Plagiomnium affine s. lat.).

CLUSTER 2. Tree layer of this community is built up of Alnus glutinosa, primary accompanied by Salix fragilis, Fraxinus excelsior and rarely by Alnus incana. Except for younger tree individuals, the species-rich shrub layer is formed mainly by Acer campestre, Corylus avellana, Euonymus europaeus, Padus avium and Sambucus nigra. The variability of dominant species (Brachypodium sylvaticum, Galeobdolon luteum agg., Urtica dioica) results in heterogeneous physiognomy of the herb layer. A large group of forest mesophilous and nitrophilous taxa (Athyrium filix-femina, Circaea lutetiana, Galium aparine, Geum urbanum, Glechoma hederacea agg., Stachys sylvatica), several elements having diverse moisture requirements (Caltha palustris, Festuca gigantea, Filipendula ulmaria, Lycopus europaeus, Lysimachia nummularia, Ranunculus repens, Stellaria nemorum) and spring species (Carex remota, Chrysosplenium alternifolium) frequently appear in the understorey. Bryophytes such as Brachythecium rivulare, Oxyrrhynchium hians and Plagiomnium undulatum exhibit also higher constancy.

CLUSTER 3. Moderately closed canopy structure of these forests consists of Alnus glutinosa and A. incana with admixture of Picea abies. An analogous species combination creates the shrub layer. The stands are clearly delimited by acidophilous species (Luzula pilosa, Vaccinium myrtillus, V. vitis-idaea) and diverse cryptogamic flora showing high fidelity to this vegetation type (Calypogeia integristipula, Chiloscyphus polyanthos, Climacium dendroides, Dicranum scoparium, Rhytidiadelphus triquetrus, Sphagnum centrale). The herb layer is further characterised by the occurrence of fen's and spring fed area's taxa (Cardamine amara agg., Carex echinata, Chaerophyllum hirsutum, Chrysosplenium alternifolium, Crepis paludosa, Galium uliginosum, Stellaria alsine, Valeriana dioica agg.) and common hygrophilous plants (Agrostis stolonifera agg., Caltha palustris, Lysimachia vulgaris, Myosotis scorpioides agg., Ranunculus repens); several of them reach high cover values. Higher frequency is typical for both species indicating submontane character (Calamagrostis villosa, Equisetum sylvaticum, Oxalis acetosella, Picea abies) and oligotrophic-tolerant mosses (Polytrichastrum formosum and Rhizomnium punctatum).

CLUSTER 4. These three-layered grey alder stands are dominated by Alnus incana with significant proportion of Picea abies. In addition to saplings of overstorey woody species, Alnus glutinosa and Frangula alnus are crucial determinants of the shrub layer. The presence of Equisetum palustre and Gentiana asclepiadea differentiates these forests from other alder vegetation. Forest mesophilous and fen species are less abundant (Tab. 1). Moreover, the peculiar aspect is driven by the presence of spring taxa (Cardamine amara agg., Chaerophyllum hirsutum, Chrysosplenium alternifolium) and numerous submontane elements (Alnus incana, Calamagrostis villosa, Equisetum sylvaticum, Gentiana asclepiadea, Oxalis acetosella, Paris quadrifolia, Picea abies, Senecio nemorensis agg.). The set of constant species involves plants of wet soils (Caltha palustris, Crepis paludosa, Filipendula ulmaria, Geum rivale, Myosotis scorpioides agg., Ranunculus repens) and clump-forming grass Deschampsia cespitosa. Ferns and nitrophytes (Athyrium filixfemina, Dryopteris carthusiana agg., Rubus idaeus, Urtica dioica) also grow in this community. Markedly developed moss layer is built up of Climacium dendroides, Dicranum scoparium, Plagiomnium affine s. lat., Polytrichastrum formosum and Rhizomnium punctatum. 
CLUSTER 5. The uniform tree layer is composed by the dominant Alnus incana with admixture of Picea abies and Salix fragilis. Other woody species (e.g. Acer pseudoplatanus) are less frequent. The same species recorded in the overstorey accompanied by Padus avium also appear in the shrub layer, usually lacking an obvious dominant. The species-rich understorey and clear floristic delimitation are caused by the presence of montane species (e.g. Aconitum variegatum, Petasites hybridus, Ranunculus lanuginosus, Senecio nemorensis agg., Silene dioica) and nitrophilous plants (Aegopodium podagraria, Carduus personata, Geranium phaeum, G. robertianum, Heracleum sphondylium, Urtica dioica). An abundant group of forest mesophytes (Asarum europaeum, Astrantia major, Daphne mezereum, Lamium maculatum, Milium effusum, Poa nemoralis, Stachys sylvatica, Stellaria nemorum) is an important structural component of these stands. Besides the constantly present taxa (Dactylis glomerata agg., Primula elatior, Roegneria canina, Valeriana officinalis agg.), the common hygrophilous and spring species contribute to the overall floristic variability (Tab. 1). Mosses related to shadow habitats with medium moist to swamp soils, such as Brachythecium rivulare, Oxyrrhynchium hians, Plagiomnium affine s. lat. and P. undulatum, show a high frequency.

\section{Environmental gradients}

The first DCA axis (Fig. 3) reflected the gradient from the eutrophic and mesotrophic forests at lower altitudes (cluster $1,2)$ to the oligotrophic vegetation of the acidic sites at higher altitudes (cluster 3 ). The main gradient was positively correlated with altitude $(r=0.89)$ and negatively with $E I V$ for nutrients $(r=-0.85)$ and soil reaction $(r=-0.76)$. Along the second DCA axis, which showed the strongest correlations with EIV for moisture $(r=-0.77)$ and light $(r=-0.55)$, the clusters were arranged from the forests of moist habitats (cluster 5) to those related to permanently waterlogged soils (cluster 1, 3). Multiple comparisons stressed the role of the same environmental drivers (Tab. 2). The individual clusters statistically differed $(P<0.05)$ especially in EIV for nutrients. Forest stands of cluster 2 were related to the nutrient-rich sites (EIV mean value 6.33), whereas the vegetation of cluster 3 exhibited an oligotrophic character (4.62). The highest moisture requirements were observed for cluster 1 (7.35) which also showed the most favorable light conditions (5.91). Although clusters 3-5 did not significantly differ in EIV for temperature (4.46-4.85), the mentioned values confirmed their preference for submontane and/or montane areas.

\section{Syntaxonomical interpretation and chorology of plant communities}

According to the list of diagnostic taxa and the comparative analysis of the species composition in the synoptic table (Tab. 1), the individual clusters can be assigned to the previously described vegetation units of the alder carr forest vegetation (Alnion glutinosae) and the broad-leaved floodplain forests (Alnion incanae). For their classification, we propose a syntaxonomical scheme with five floristically and ecologically interpretable associations: Carici elongatae-Alnetum glutinosae s. lat. (cluster 1; alder carr forests growing on permanently waterlogged soils), Stellario-Alnetum glutinosae (cluster 2; riparian alder vegetation on mesic to humid sites along small brooks), Piceo-Alnetum (cluster 3; submontane and montane oligotrophic spruce-alder forests on waterlogged habitats), Cardamino amarae-Alnetum incanae (cluster 4; grey alder stands in spring fed areas) and Alnetum incanae (cluster 5; submontane

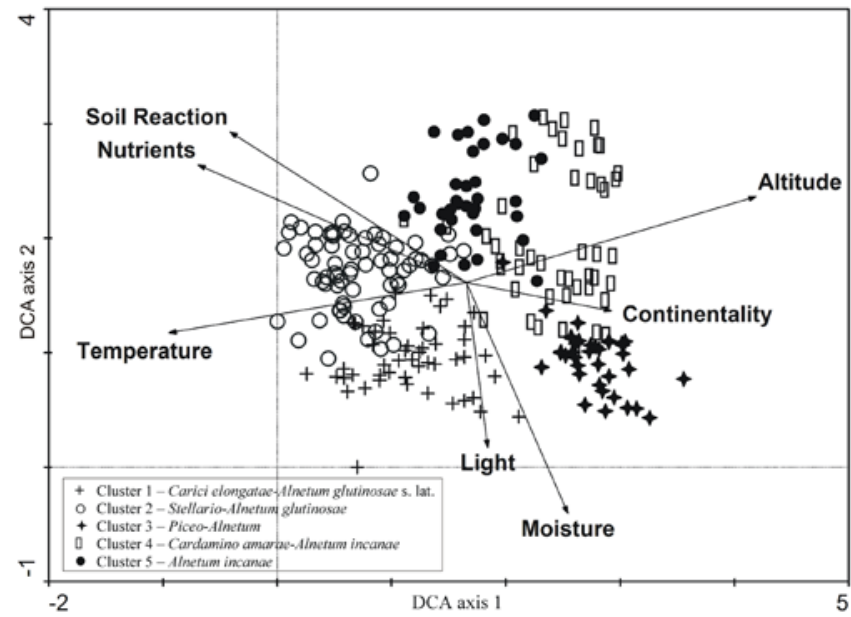

Fig. 3 Biplot of alder vegetation relevés based on DCA with passive projection of environmental variables (altitude and EIV). The length of the first axis gradient is 3.556; the first two ordination axes explain $7.3 \%$ and $50.8 \%$ of the species data and species-environment relation, respectively. Pearson correlation coefficients with the first two DCA axes $\left({ }^{\star} P<0.05\right.$; ns: $\left.P>0.05\right)$ : altitude $\left(0.89^{*} ; 0.24^{*}\right)$, light $(0.08 \mathrm{~ns}$; $\left.-0.55^{\star}\right)$, temperature $\left(-0.92^{\star} ;-0.12^{\star}\right)$, continentality $\left(0.47^{\star} ;-0.09 \mathrm{~ns}\right)$, moisture $\left(0.34^{\star} ;-0.77^{\star}\right)$, soil reaction $\left(-0.76^{\star} ; 0.50^{\star}\right)$, nutrients $\left(-0.85^{\star} ; 0.41^{\star}\right)$.

and montane streamside grey alder forests on mesic sites). Their syntaxonomical affiliation and synonyms are:

Class: Alnetea glutinosae Br.-Bl. Et R. Tx. ex Westhoff et al. 1946

Order: Alnetalia glutinosae R. Tx. 1937

Alliance: Alnion glutinosae Malcuit 1929

Ass. Carici elongatae-Alnetum glutinosae Schwickerath 1933 Synonyms: Carici elongatae-Alnetum glutinosae Koch 1926 (Art. 2b), Alnus glutinosa-Dryopteris spinulosa-Ass. Klika 1940 (syntax. syn.), Alnus glutinosa-Molinia coerulea Šmarda 1951 (Art. 3d), Cariceto elongatae-Alnetum medioeuropaeum (Koch 1926) R. Tx. et Bodeux 1955 (Art. 34a), Carici elongataeAlnetum glutinosae boreale Preising et Bodeux 1955 (Art. 34a), Caltho palustris-Alnetum glutinosae Šomšák 1961 p.p. (syntax. syn.)

Class: Querco-Fagetea Br.-Bl. et Vlieger in Vlieger 1937

Order: Fagetalia Pawłowski in Pawłowski et al. 1928

Alliance: Alnion incanae Pawłowski in Pawłowski et al. 1928

Suballiance: Alnenion glutinoso-incanae Oberd. 1953

Ass. Stellario-Alnetum glutinosae Lohmeyer 1957

Synonyms: Querceto-Carpinetum alnetosum Mikyška 1939 (syntax. syn.), Caltho palustris-Alnetum glutinosae Šomšák 1961 p.p. (syntax. syn.)

Ass. Piceo-Alnetum Mráz 1959

Ass. Cardamino amarae-Alnetum incanae Šomšák 1961

Ass. Alnetum incanae Lüdi 1921

Synonyms: Alnetum incanae Aicher et Siegrist 1930 (syntax. syn.), Alnetum incanae carpaticum Klika 1936 (Art. 34a), Alnetum incanae boreocarpaticum Jurko 1961 (Art. 34a).

The vegetation analysis demonstrated several geographical regularities of alder-dominated forests. Except for alder carrs of the Carici elongatae-Alnetum glutinosae s. lat., which occupy suitable habitats equally all over the study area, the remaining vegetation types of Alnenion glutinoso-incanae suballiance have 
Tab. 2 Means and standard deviations for altitude and Ellenberg indicator values (EIV).

\begin{tabular}{|c|c|c|c|c|c|}
\hline Number of cluster & 1 & 2 & 3 & 4 & 5 \\
\hline Altitude $(\mathrm{m})^{*}$ & $426.16 \pm 124.75^{a}$ & $390.23 \pm 136.79^{\text {a }}$ & $843.11 \pm 59.37^{b}$ & $826.15 \pm 118.05^{b}$ & $743.00 \pm 131.17^{b}$ \\
\hline EIV_Light & $5.91 \pm 0.27^{\mathrm{c}}$ & $5.37 \pm 0.37^{\mathrm{a}}$ & $5.69 \pm 0.37^{b c}$ & $5.41 \pm 0.38^{\mathrm{a}}$ & $5.48 \pm 0.30 \mathrm{ab}$ \\
\hline$E I V \_$Temperature* & $5.17 \pm 0.17^{\mathrm{a}}$ & $5.26 \pm 0.13^{a}$ & $4.46 \pm 0.21^{\mathrm{b}}$ & $4.51 \pm 0.25^{\mathrm{b}}$ & $4.85 \pm 0.20^{\mathrm{c}}$ \\
\hline EIV_Continentality ${ }^{*}$ & $3.73 \pm 0.15 \mathrm{abc}$ & $3.67 \pm 0.14^{\mathrm{a}}$ & $3.90 \pm 0.24^{\mathrm{de}}$ & $3.86 \pm 0.16^{\mathrm{ce}}$ & $3.79 \pm 0.11^{\mathrm{acd}}$ \\
\hline EIV_Moisture & $7.35 \pm 0.32^{b}$ & $6.44 \pm 0.38^{\text {a }}$ & $7.25 \pm 0.39^{b}$ & $6.75 \pm 0.39^{c}$ & $6.49 \pm 0.35^{\mathrm{a}}$ \\
\hline EIV_Soil_Reaction* & $6.26 \pm 0.37^{a}$ & $6.57 \pm 0.19^{b}$ & $4.91 \pm 0.39^{c}$ & $5.89 \pm 0.49^{\mathrm{a}}$ & $6.63 \pm 0.24^{b}$ \\
\hline$E I V \_$Nutrients $(\wedge 4)$ & $5.82 \pm 0.37^{a}$ & $6.33 \pm 0.31^{b}$ & $4.62 \pm 0.49^{c}$ & $5.39 \pm 0.47^{\mathrm{d}}$ & $6.00 \pm 0.24^{\mathrm{a}}$ \\
\hline
\end{tabular}

Significant differences among particular clusters (vegetation types) in one-way ANOVA and post-hoc Tukey HSD test or (marked with “‘”) Kruskal-Wallis ANOVA $(P<0.05)$ are displayed by different letters $(\mathrm{a}-\mathrm{e}) . \wedge 4-$ biquadratic transformation.

partially limited distribution along the latitudinal gradient (Fig. 4). Within the distribution range of the riparian forests, the ecological characteristics of their dominant tree species and overall community structure indicated higher concentration of Alnetum incanae in northern relatively high-altitudinal areas. The Piceo-Alnetum and Cardamino amarae-Alnetum incanae associations belong to the most scarce alder vegetation in the central Slovakia. All available phytosociological relevés of spruce-alder forests have been collected only in the Podtatranské kotliny basins (Fig. 4).

\section{Discussion}

Classification and diversity of Central European alder forests result from the variability of the species composition, which is controlled by the nutrient and moisture heterogeneity along with temperature and altitude (Fig. 3). The significant influence of analogous environmental gradients on structure and composition of this vegetation has already been proven $[4,43,44]$. In addition to the just-discussed effect of distinctive site conditions, existence of alder forests is also a matter of the recent land use, secondary succession, drainage, melioration and afforestation of permanently wet meadows $[45,46]$. Ecological conditions of alder carr (Alnion glutinosae) and riparian alder forests (Alnion incanae) are relatively constantly interpreted in scientific literature, but unfortunately their syntaxonomy seems to be much more complicated regarding the accurate application of the nomenclatural rules, vague syntaxonomical position of the numerous associations or confused use of the communities names.

\section{Alder carr forests}

The floristic and ecological delimitation of alder carr forests is most likely determined by conspicuous micro-relief character with waterlogged hollows and drier hummock $[2,11]$. This environmental heterogeneity is emphasized by a high groundwater table and swampy soils that are generally rich in organic substrate [43]. The first European comprehensive synthesis of the Alnion glutinosae phytosociological data was done by Bodeux [3] who distinguished four associations and provided their diagnostic features based on the geographic pattern. The majority of later classification systems reflected the soil nutrient/acidity complex and hydrological regime rather than the geographic gradient (e.g. $[4,16,47])$, as they appeared to be better predictors of vegetation variability within the alder carr forests. The Alnion glutinosae stands were documented only with Carici elongatae-Alnetum glutinosae in central Slovakia (Tab. 1). This meso- to eutrophic community was originally described as an association by Schwickerath [34] from the western Germany. In accord with some syntaxonomical revisions $[16,26]$, we used the broader defined concept of this association. There are a few relevés that were previously ascribed to the Carici acutiformis-Alnetum glutinosae association in the same geographical area [24]. The unsupervised method of numerical classification supported by a relative similarity in overall species composition did not create separate cluster of this vegetation type. These relevés were thus merged into the Carici elongatae-Alnetum glutinosae s. lat. in our analysis (cluster 1; Tab. 1, Fig. 3). Nevertheless, these stands differ in the diagnostic species, physiognomy and habitat conditions at the local scale [24]. The Carici elongatae-Alnetum glutinosae has recently been documented in lowlands and submontane areas of various European countries $[4,5,14,15,17,44]$. In Poland, plant communities formerly classified within this syntaxon $[8,48]$ were revised by Solińska-Górnicka [11]. This author suggested its splitting into Ribo nigri-Alnetum Solińska-Górnicka (1975) 1987 and Sphagno squarrosi-Alnetum Solińska-Górnicka (1975) 1987 associations based on significant differences in their species composition, ecology and chorology. The general conclusions of many national vegetation overviews (e.g. $[4,11])$ indicated that the relevés of various associations quoted in partial local revisions must be analyzed at a broad geographic scale in the future. Scarcity and unavailability of vegetation studies have been frequently reported as the substantial constraints of a comprehensive synthesis, but the development of national phytosociological databases [49] allows performing comparative studies covering several regions.

Alder carr forests are generally characterized by a high diversity and variability of understorey species composition and by presence of transitional communities. These features often led to new proposals for delimitation or subdivisions of vegetation units in the past. However, several of them had not been differentiated in accordance with ICPN [42]. In central Slovakia, Šomšák [20] described an association Caltho-Alnetum Šomšák 1961 from the Slovenské rudohorie Mts., but there is no nomenclatural type relevé (holotype; ICPN Art. 19) given in that publication. For this reason, we have designated here a lectotype from relevés published in the original diagnosis [20] (table 6, relevé No. 6, lectotypus hoc loco). Since this data matrix contains only the species Caltha palustris in the herb layer and dominant tree species Alnus glutinosa in the overstorey, we 
a)
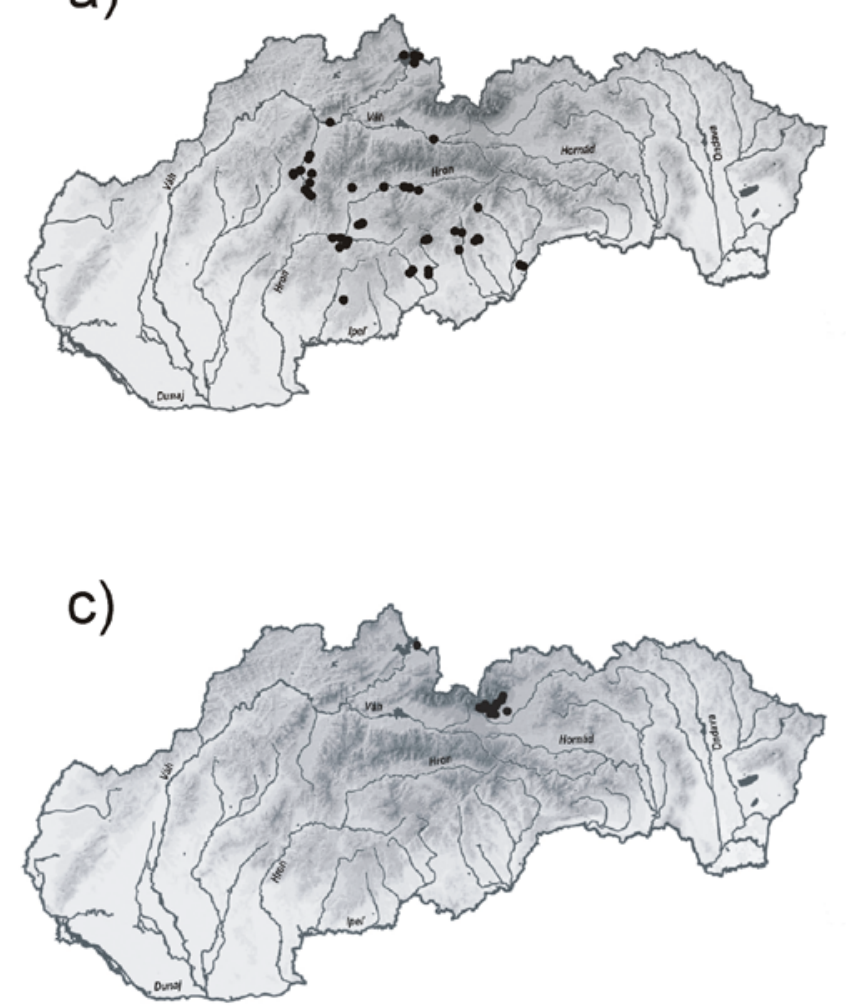

b)

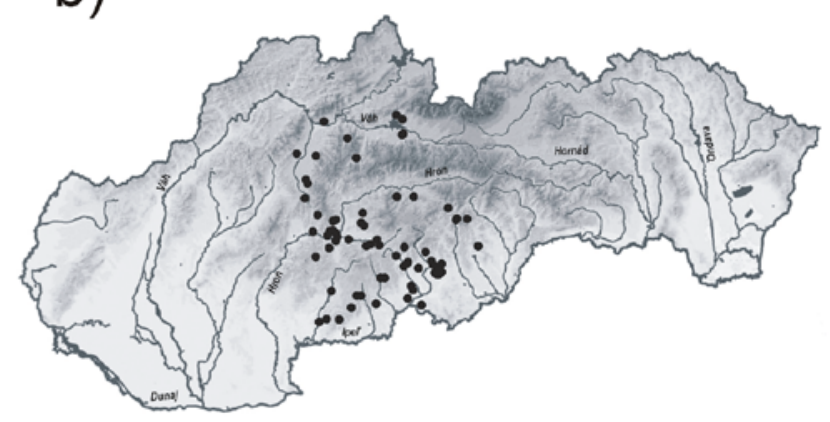

d)

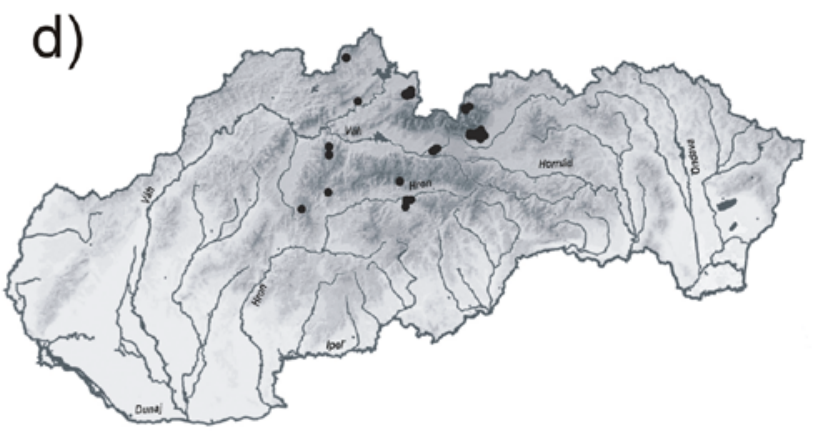

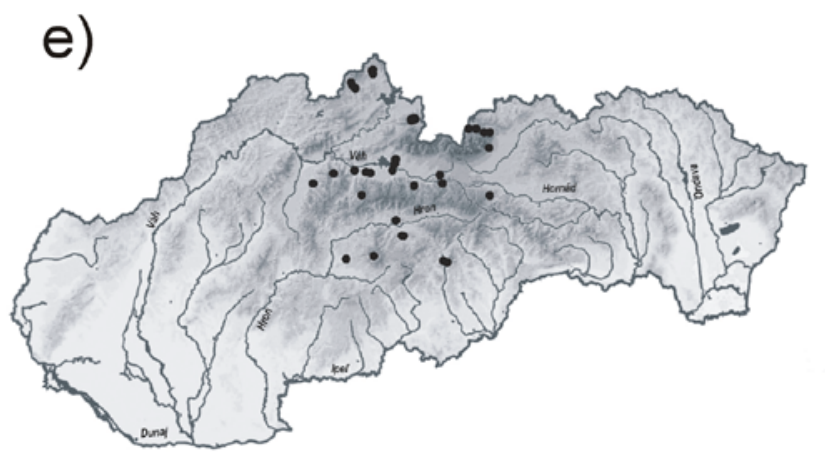

Fig. 4 Spatial distribution of vegetation units with dominance of alder species in central Slovakia. a Carici elongatae-Alnetum glutinosae s. lat. (cluster 1). b Stellario-Alnetum glutinosae (cluster 2). c Piceo-Alnetum (cluster 3). d Cardamino amarae-Alnetum incanae (cluster 4). e Alnetum incanae (cluster 5).

have added epithets (ICPN Recomm. 10C) and interpreted the name as Caltho palustris-Alnetum glutinosae Šomšák 1961. The phytosociological material includes very different relevés with heterogeneous species composition and therefore the relevé set can be assigned not only to the alder carr forests but also to the riparian alder vegetation. If this floristic variability along with habitat characteristics and understorey composition of the original diagnosis $[34,35]$ are taken into account, the name Caltho palustris-Alnetum glutinosae Šomšák 1961 thus becomes a syntaxonomic synonym for a part of the associations Carici elongatae-Alentum glutinosae and Stellario-Alnetum glutinosae. The name Caltho-Alnetum Šomšák 1961 was later modified by Šomšák [50] as Caltho laetae-Alnetum glutinosae (Šomšák 1961) comb. nova, however the choice of the nomenclatural type from the data matrix published in 1979 [50] (table 1, relevé No. 10, neotypus) and not from former paper [20] is considered to be invalid (ICPN Art. 19). The additional taxonomic determination of Caltha palustris subsp. laeta [50] resulted in an unambiguous assignment of this taxon by the original author to populations recorded in relevés from the Slovenské rudohorie Mts. as well. In this sense, it is possible to apply the name Caltho laetaeAlnetum glutinosae for the community originally described as Caltho-Alnetum Šomšák 1961 (ICPN Recomm. 10C). The Caltho laetae-Alnetum glutinosae Šomšák (1961) 1979 is therefore a younger homonym of the Caltho palustris-Alnetum glutinosae Šomšák 1961, i.e. an illegitimate name according to ICPN Art. 31. In addition, there was observed an evident shift by ecological interpretation of this community from hygrophilous forests with numerous mesotrophic species to hygrophilous ones with regular occurrence of acidophytes occupying hemioligotrophic habitats [50]. However, the species composition of the relevés from the High Tatras Mts. [50] corresponds to the alder vegetation of Piceo-Alnetum (Alnion incanae). The previous investigation gave an ambiguous answer concerning 
its position in the classification system. Formerly, it had been classified in the Alnion glutinosae alliance [26]. Regarding the overall floristic spectrum, ecological requirements and particularly the results of our analysis (Tab. 1, Fig. 3), we propose its subordination into the Alnion incanae forests.

\section{Riparian alder forests}

The present findings of the variability in environmental conditions and species composition affecting the internal differentiation of riparian forests (Tab. 1, Tab. 2) are in agreement with the outcomes of other studies (e.g. $[4,16])$. Four associations were distinguished using the numerical classification, including mesic riparian alder vegetation on habitats from the colline to the montane belt, oligotrophic spruce-alder forests and springalder vegetation. Forest stands with a similar floristic structure and ecology are known from several Central European countries $[4,7,10,13,17]$. The analysis did not form a separate cluster for the Carici remotae-Fraxinetum association. The stands with the species composition similar to these eutrophic spring ash-alder forests $[9,12]$ had marginal occurrence in the study area, and they were recorded only in a few relevés. Considerable floristic variability and a small number of relevés in the data set most likely resulted in their assignment into the Stellario-Alnetum glutinosae association (cluster 2; Tab. 1).

The general arrangement of the Alnion incanae alliance consists of the commonly accepted syntaxa with obvious diagnostic features and a wider geographical range (Stellario-Alnetum glutinosae, Alnetum incanae, Fraxino-Alnetum W. Matuszkiewicz 1952), and the plant communities with a specific local floristic combination, e.g. Cardamino amarae-Alnetum incanae in Slovakia [20], Paridi quadrifoliae-Alnetum glutinosae Kevey in Borhidi et Kevey 1996 in Hungary [15], Carici rostrataeAlnetum incanae Karner in Willner et Grabher 2007 in Austria [16] or Ornithogalo pontici-Alnetum glutinosae Didukh 1996 em. Onyshchenko 2009 in Ukraine [19]. The syntaxonomical and nomenclatural stability is thus probably dependent on the comparison of large-scale phytosociological data set, which might lead to the reduction of some associations to synonyms. For example, the forest stands reported from the Eastern Carpathians as Caltho laetae-Alnetum (Zarzycki 1963) Stuchlik 1968 $[51,52]$ and alder vegetation in the foothills of the High Tatras Mts. [50] correspond to the Piceo-Alnetum association, what is consistent with the revision of the Ukrainian Fagetalia sylvaticae forests, where Caltho laetae-Alnetum (Zarzycki 1963) Stuchlik 1968 has been quoted in the synonym list of the latter one [19].

The syntaxonomical and nomenclatural evaluation of particular clusters indicated an incorrect use of the authorship for the name Piceo-Alnetum Rubner ex Oberdorfer 1957. The spruce-alder forests with strictly mesotrophic character were for the first time described by Rubner [53] as Piceeto-Alnetum glutinosae without any abundance-cover values for individual plant species, and therefore it must be regarded as invalid (ICPN Art. 2b). In the syntaxonomic scheme proposed by Oberdorfer [54] for the alliance Alno-Ulmion Br.-Bl. et Tx. 43, there was reported a synoptic table with a range of constancy classes for the recorded species, which can analogically be considered as an insufficient validation in term of ICPN Art. 2 (see also [4,9]). The Piceo-Alnetum was thus validly published by Mráz [36] from the Czech Republic, who used this name for the oligotrophic spruce-alder forests on waterlogged soils. Outside the Czech Republic, it has been found in Ukraine [19] and analogous community has also been mentioned under various names from Germany [17], Austria [16] and Poland [55].
The species composition of the alder forests seems to be relatively homogeneous, with a number of species able to grow across different scales and areas together with a set of species reflecting the local environmental gradients or phytogeographical influences. These floristic patterns allow us to distinguish the associations distributed under similar conditions throughout the Central Europe and, at the same time, forests with partial preference to a specific geographic region. In Slovakia, the forest vegetation dominated by Alnus glutinosa or A. incana shows a good coincidence with analogous communities recognized in the most recent national vegetation overviews, which are traditionally classified in the Alnion glutinosae and Alnion incanae alliances. Moreover, the current study unifies the previous local classification systems and represents an important step towards the national synthesis of alder forests.

\section{Acknowledgments}

We would like to thank J. Kliment and M. Chytrý for advice in solving nomenclature issues, J. Douda for valuable comments on previous draft of this manuscript and D. Kúdelová for language improvement. The authors are also grateful to A. Almášiová and K. Sládeková for providing difficult accessible literature. The research was supported by the Science Grant Agency of the Ministry of Education of the Slovak Republic and the Slovak Academy of Sciences (VEGA 2/ 0059/ 11, VEGA 2/ 0027/ 13) and by the Grant Agency of Faculty of Education in CU (GAPF 1/ 24/ 2012).

\section{Authors' contributions}

The following declarations about authors' contributions to the research have been made: conceived the idea, analyzed the data and led the writing: MS, RH; identified herbarium specimens of bryophytes: AP; collected data in the field and commented on the manuscript: MS, RH, AP, DD.

\section{References}

1. Carnicer J, Brotons L, Stefanescu C, Peñuelas J. Biogeography of species richness gradients: linking adaptive traits, demography and diversification. Biol Rev. 2012;87(2):457-479. http://dx.doi. org/10.1111/j.1469-185X.2011.00210.x

2. Klika J. Die Pflanzengesellschaften des Alnion-Verbandes. Preslia. 1940;18-19:97-112.

3. Bodeux A. Alnetum glutinosae. Mitt Florist-Soziol Arbsgem. 1955;5:114-137.

4. Douda J. Formalized classification of the vegetation of alder carr and floodplain forests in the Czech Republic. Preslia. 2008;80:199-224.

5. Sburlino G, Poldini L, Venanzoni R, Ghirelli L. Italian black alder swamps: their syntaxonomic relationships and originality within the European context. Plant Biosyst. 2011;145(1 suppl):148-171. http://dx.doi.org/10.1 080/11263504.2011.602746

6. Meusel H, Jäger E, Weinert E. Vergleichende Chorologie der Zentraleuropäischen Flora. Jena: Gustav Fischer Verlag; 1965.

7. Ellenberg H. Vegetation Mitteleuropas mit den Alpen. Stuttgart: Ulmer; 1982.

8. Matuszkiewicz W, Traczyk H, Traczyk T. Materialy do fitosocjologicznej systematyki zespołów olsowych w Polsce. Acta Soc Bot Pol. 1958;27:21-44.

9. Moravec J, Husová M, Neuhäusl R, Neuhäuslová-Novotná Z. Die 
Assoziationen mesophiler und hygrophiler Laubwälder in der Tschechischen Sozialistischen Republik. Prague: Academia; 1982. (Vegetace ČSSR; vol 12).

10. Schwabe A. Monographie Alnus incana-reicher Waldgesellschaften in Europa Variabilität und Ähnlichkeiten einer azonal verbreiteten Gesellschaftsgruppe. Phytocoenologia. 1985;13:197-302.

11. Solińska-Górnicka B. Alder (Alnus glutinosa) carr in Poland. Tuexenia. 1987;7:329-346.

12. Oberdorfer E. Der europäische Auenwald. Naturk Forsch SüdwestDeutschl. 1953;12:23-70.

13. Matuszkiewicz W. Przewodnik do oznaczania zbiorowisk roślinnych Polski. Warsaw: Polish Scientific Publishers PWN; 2008.

14. Solomakha VA. The syntaxonomy of vegetation of Ukraine. Ukr Phytosociol Collection A. 1996;4(5):1-121.

15. Kevey B. Magyarország erdötársulásai (Forest associations of Hungary). Tilia. 2008;14:1-489.

16. Willner W, Grabherr G. Die Wälder und Gebüsche Österreichs: Ein Bestimmungswerk mit Tabellen. Heidelberg: Spektrum Akademischer Verlag; 2007.

17. Pott R. Die Pflanzengesellschaften Deutschlands. Stuttgart: E. Ulmer Verlag; 1992

18. Jarolímek I, Šibík J, Hegedüšová K, Janišová M, Kliment J, Kučera P, et al. A list of vegetation units of Slovakia. In: Jarolímek I, Šibík J, editors. Diagnostic, constant and dominant species of the higher vegetation units of Slovakia. Bratislava: Veda; 2008. p. 295-329.

19. Onyshchenko VA. A revised classification of Ukrainian forests of the order Fagetalia sylvaticae. Tuexenia. 2010;30:31-45.

20. Šomšák L. Jelšové porasty Spišsko-gemerského rudohoria. Acta Fac Rer Natur Univ Comenianae Bot. 1961;6:407-459.

21. Kliment J, Watzka R. Lesné spoločenstvá Drienčanského krasu. In: Kliment J, editor. Príroda Drienčanského krasu. Banská Bystrica: Štátna ochrana prírody SR; 2000. p. 191-214.

22. Berta J. Waldgesellschaften und Bodenverhältnisse in der Theisstiefebene. Bratislava: Slowakischen Akademie der Wissenschaften.; 1970. (Vegetácia ČSSR; vol 1).

23. Kontriš J. Pôdnoekologické a fytocenologické pomery lužných lesov Liptovskej kotliny. Biol Práce Slov Akad Vied. 1981;27(1):1-164.

24. Slezák M, Hrivnák R, Petrášová A. Syntaxonomy and ecology of black alder vegetation in the southern part of central Slovakia. Hacquetia. 2011;10(2):119-136. http://dx.doi.org/10.2478/v10028-011-0006-6

25. Jurko A. Das Alnetum incanae in der Mittelslowakei (II. Die Auenwälder in den Westkarpaten). Biológia. 1961;16:321-339.

26. Šomšák L. Alnion glutinosae Malcuit 1929 na Slovensku (Západné Karpaty). Acta Fac Rer Natur Univ Comenianae Bot. 2000;40:81-102.

27. Hegedüšová K. Centrálna databáza fytocenologických zápisov (CDF) na Slovensku. Bull Slov Bot Spoločn. 2007;29:124-129.

28. Westhoff V, van der Maarel E. The Braun-Blanquet approach. In: Whittaker RH, editor. Ordination and classification of communities. Hague: Junk; 1973. p. 617-727.

29. Barkman JJ, Doing H, Segal S. Kritische Bemerkungen und Vorschläge zur quantitativen Vegetationsanalyse. Acta Bot Neerl. 1964;13:394-419.

30. McCune B, Mefford MJ. PC-ORD: Multivariate analysis of ecological data: version 4.0. Gleneden Beach: MjM Software Design; 1999.

31. Botta-Dukát Z, Chytrý M, Hájková P, Havlová M. Vegetation of lowland wet meadows along a climatic continentality gradient in Central Europe. Preslia. 2005;77:89-111.

32. Tichý L. JUICE, software for vegetation classification. J Veg Sci. 2002;13(3):451-453. http://dx.doi.org/10.1111/j.1654-1103.2002.tb02069.x

33. Lüdi W. Die Pflanzengesellschaften des Lauterbrunnentales und ihre Sukzession: Versuch zur Gliederung der Vegetation eines Alpentales nach genetisch-dynamischen Gesichtpunkten. Beitr Geobot Landesaufn Schweiz. 1921;9:1-364.

34. Schwickerath M. Die Vegetation des Landkreises Aachen und ihre Stellung im nördlichen West-Deutschland. Aachen Beitr Heimatk. 1933;13:1-135.

35. Lohmeyer W. Der Hainmieren-Schwarzerlenwald [Stellario-Alnetum glutinosae (Kästner 1938)]. Mitt Florist-Soziol Arbsgem. 1957;6-7:247-257.

36. Mráz K. Př́íspevek k poznání původnosti smrku a jedle ve vnitrozemí Čech. Práce Výzk Úst Lesn. 1959;17:135-180.

37. Chytrý M, Tichý L, Holt J, Botta-Dukát Z. Determination of diagnostic species with statistical fidelity measures. J Veg Sci. 2002;13(1):79-90. http:// dx.doi.org/10.1111/j.1654-1103.2002.tb02025.x

38. Ellenberg H, Weber HE, Düll R, Wirth W, Werner W, Paulißen D. Zeigerwerte von Pflanzen in Mitteleuropa. Scr Geobot. 1992;18:1-258.

39. Marhold K, Hindák F, editors. Zoznam nižších a vyšších rastlín Slovenska. Bratislava: Veda; 1998.

40. Hill MO, Bell N, Bruggeman-Nannenga MA, Brugués M, Mefford MJ, Enroth J, et al. An annotated checklist of the mosses of Europe and Macaronesia. J Bryol. 2006;28(3):198-267. http://dx.doi. org/10.1179/174328206X119998

41. Söderström L, Urmi E, Váña J. The distribution of Hepaticae and Anthocerotae in Europe and Macaronesia - update 1-427. Cryptogamie Bryologie. 2007;28:299-350.

42. Weber HE, Moravec J, Theurillat JP. International code of phytosociological nomenclature. 3rd edition. J Veg Sci. 2000;11(5):739-768. http://dx.doi. org $/ 10.2307 / 3236580$

43. Döring-Mederake U. Alnion forests in Lower Saxony (FRG), their ecological requirements, classification and position within Carici elongatae-Alnetum of northern Central Europe. Vegetatio. 1990;89(2):107-119. http://dx.doi. org/10.1007/BF00032164

44. Prieditis N. Alnus glutinosa-dominated wetland forests of the Baltic Region: community structure, syntaxonomy and conservation. Plant Ecol. 1997;129(1):49-94. http://dx.doi.org/10.1023/A:1009759701364

45. Douda J, Čejková A, Douda K, Kochánková J. Development of alder carr after the abandonment of wet grasslands during the last 70 years. Ann For Sci. 2009;66(7):712-712. http://dx.doi.org/10.1051/forest/2009065

46. Orczewska A. The impact of former agriculture on habitat conditions and distribution patterns of ancient woodland plant species in recent black alder [Alnus glutinosa (L.) Gaertn.] woods in south-western Poland. For Ecol Manage. 2009;258(5):794-803. http://dx.doi.org/10.1016/j. foreco.2009.05.021

47. Neuhäuslová Z. Alnetea glutinosae. In: Moravec J, editor. Přehled vegetace České republiky, Svazek 4. Vrbotopolové luhy a bažinné olšiny a vrbiny. Prague: Academia; 2003. p. 41-57.

48. Marek S. Biologia i stratygrafia torfowisk olszynowych w Polsce. Zesz Probl Post Nauk Rol. 1965;57:1-264.

49. Schaminée JHJ, Hennekens SM, Chytrý M, Rodwell JS. Vegetation-plot data and databases in Europe: an overview. Preslia. 2009;81:173-185.

50. Šomšák L. Torfwälder fluvioglazialen Abagerungen der Hohen Tatra. Acta Fac Rer Natur Univ Comenianae Bot. 1979;27:1-37.

51. Zarzycki K. Lasy Bieszczadów Zachodnich. Acta Agr Silv Ser Silv. 1963;3:1-132.

52. Stuchlik L. Zbiorowiska leśne i zaroślowe pasma Policy w Karpatach Zachodnich. Fragm Flor Geobot. 1968;14(4):441-484.

53. Rubner K. Die Roterlengesellschaft der oberbayerischen Grundmoräne. Forstarchiv. 1954;25(6):137-142.

54. Oberdorfer E. Süddeutsche Pflanzengesellschaften. Pflanzensoziologie. 1957;10:1-564.

55. Koczur A. Zróżnicowanie siedliskowe bagiennej olszyny górskiej Caltho laetae-Alnetum (Zarz. 1963) Stuchlik 1968 v Babiogórskim Parku Narodowym. Sylwan. 2011;155:112-119.

56. Hrivnák R, Cvachová A. Vegetácia zátopovej oblasti projektovanej vodárenskej nádrže Hronček v doline Kamenistého potoka vo Veporských vrchoch. Acta Fac Forest. 1999;51:11-27.

57. Hrivnák R, Kochjarová J, Blanár D, Šoltés R. Jelšové lesy na Muránskej planine - zrhnutie súčasných fytocenologických poznatkov. Reussia. 2009;5(1-2):23-33. 
58. Miadok D. Vegetácia severozápadnej časti Gemerského rudohoria $[\mathrm{PhD}$ thesis]. Bratislava: Comenius University in Bratislava; 1974.

59. Slezák M, Letz DR, Hrivnák R, Vlčko J, Turis P, Blanár D. Aktuálne poznatky o výskyte niektorých zriedkavejších cievnatých rastlín na území stredného Slovenska. Bull Slov Bot Spoločn. 2012;34:19-44.

60. Watzka R. Spoločenstvá lužných jelšín Lubochnianskej doliny vo Vel'kej Fatre. Bull Slov Bot Spoločn. 1999;21:151-160.

61. Ferančíková V. Vegetačná mapa lesov Podtatranskej kotliny - čast’ Tatranská Polianka [Master thesis]. Bratislava: Comenius University in Bratislava; 1994.

62. Holotová E. Vegetačná mapa lesov Podtatranskej koltiny - čast' Smokovce [Master thesis]. Bratislava: Comenius University in Bratislava; 1994.

63. Matová H. Vegetačná mapa lesov Podtatranskej kotliny - čast' Tatranské Zruby [Master thesis]. Bratislava: Comenius University in Bratislava; 1994.

64. Viceníková A. Viceníková A. Lesné spoločenstvá glaciálno-fluviálnych sedimentov Podtatranskej kotliny [PhD thesis]. Bratislava: Comenius University in Bratislava; 1998.
65. Černušáková D. Fytocenologická a ekologická charakteristika lesov masívu Osobitej [PhD thesis]. Bratislava: Comenius University in Bratislava; 1983.

66. Kubíček F, Šimonovič V, Viceníková A, Mačor S. Productivity of the herb and moss layer in forest ecosystems at the nature reserve Bor (Podspády); the Tatra national park. Štúdie o TANAP-e. 1997;35(2):143-159.

67. Šomšák L. Erlenbruchwald von Bacúch (Bacúšska jelšina). Acta Fac Rer Natur Univ Comenianae Bot. 1967;15:1-11.

68. Šomšák L, Viceníková A, Mačor S. Cardamino-Alnetum incanae luecanthemetosum waldsteinii subas. nova v Podtatranskej brazed. Bull Slov Bot Spoločn. 1993;15:37-41.

69. Jurko A, Májovský J. Lužné lesy v západných Karpatoch I. Alnetum incanae na severnej Orave. Acta Fac Rer Natur Univ Comenianae Bot. 1956;8-9:363-385.

70. Kanka R. Alnetum incanae Lüdi 1921 v Belianskych Tatrách. Biosozologia. 2003;1:52-59.

71. Kučera P. Lesné spoločenstvá Belianskej doliny vo Velkej Fatre [Master thesis]. Bratislava: Comenius University in Bratislava; 2002. 